\title{
Conflictos Bioeticos y Biojuridicos en la Salud Pública Global ${ }^{1}$
}

\author{
Alina Carmen Celi Frugoni \\ Doctora en Derecho (Universidad de Alicante, Programa \\ Doctorado en Derecho Ambiental), Máster en Derecho \\ Administrativo Económico (Universidad de Montevi- \\ deo), Posgraduada en Derecho Ambiental (Universidad \\ Austral de Buenos Aires). El artículo ha sido elaborado \\ en el marco de la investigación llevada a cabo en la \\ Universidad do Rio do Vale dos Sinos (Unisinos, Brasil) \\ bajo la orientación del profesor doctor Leonel Severo \\ Rocha (Programa Nacional de Posdoctorado (Capes). \\ aceli@correo.um.edu.uy
}

\section{Resumen:}

El artículo trata sobre conflictos bioéticos y biojurídicos en el contexto de la salud pública. Si bien instituciones como la Organización Mundial de la Salud, han contribuido en gran forma para la detección, investigación y resolución de problemas en la salud humana a partir del uso de nuevas tecnologías, diferencias socio-económicas y políticas de los Estados han abierto una brecha considerable de desigualdades humanas. Por ello la importancia del abordaje inicial de esos conflictos y el reconocimiento de sus fundamentos para reorientar los sistemas jurídicos hacia la adopción de soluciones teóricas y prácticas.

Palabras-clave: Salud pública global. Bioética. Bioderecho. Tecnologías.

${ }^{1}$ Comunicación al Seminário Internacional Direitos Humanos e Democracia (Unijuí, 29-30, Octubre, 2014). 
BIOETHICAL AND BIOJURIDICAL CONFLICTS IN GLOBAL PUBLIC HEALTH

\begin{abstract}
This article deals with bioethical and biojuridical conflicts in the context of public health. While institutions such as the World Health Organization have contributed greatly to the detection, investigation and resolution of human health problems by the use of new technologies, socio- economic political differences of States have provoked considerable human inequalities. Hence the importance of the initial approach of these conflicts and the recognition of their juridical founding to reorient law systems towards the adoption of theoretical and practical solutions.
\end{abstract}

Keywords: Global Public Health. Bioethics. Biolaw. Technologies.

\title{
SUMÁRIO
}

1 Introducción. 2 Principales Problemas de la Salud Pública Global y su Relación con la Bioética y el Bioderecho. 3 Conclusiones. 4. Referencias. 


\section{INTRODUCCIÓN}

La bioética se erige actualmente como disciplina científica, pero también como respuesta de los movimientos socioculturales a los avances derivados de las nuevas tecnologías surgidas en la segunda mitad del siglo 20; fenómeno del cual se derivan las llamadas éticas aplicadas a los órdenes de la política y el medio ambiente, entre otros. ${ }^{2}$

Etimológicamente el concepto de bioética proviene del griego (bios-vida) y (ethos- ética) y en virtud del progreso médico, su debate actual se centra tanto en el ámbito académico, como en el sociocultural y político. ${ }^{3}$

Sin embargo la bioética no tiene demasiado sentido considerada como disciplina aislada de otras áreas del conocimiento. Como bien señala Guy Durant, ${ }^{4}$ esta palabra (bioética) realmente es comprendida en cuanto es vinculada a otros vocablos como por ejemplo, la moral, la ética, la deontología, y la ética biomédica. En particular en materia de salud pública, interesa destacar la relación de la bioética con la moral y con otro concepto de origen también reciente, que es el bioderecho. A su vez la moral se enlaza a las costumbres y en ese sentido a las formas de vida, ${ }^{5}$ que incluyen aquellas prácticas que llevamos a cabo para desarrollar nuestras actividades profesionales.

Observando el fenómeno del desarrollo de la bioética en las últimas décadas y desde el ámbito de la salud pública, dos son los tipos de prácticas de orden técnico y político de interés para el análisis de la cuestión:

${ }^{2}$ Carvalho Fortes, Paulo Antonio; Campos Pavone Zoboli, Elma Lourdes. Bioética e saúde pública: entre o individual e o coletivo. In: Carvalho Fortes, Paulo Antonio; Campos Pavone Zoboli, Elma Lourdes (Orgs.). Bioética e saúde pública. São Paulo: Edições Loyola, 2003. p.11-24.

${ }^{3}$ Durant, Guy. A bioética, natureza, princípios, objetivos. São Paulo: Paulus, 1995. p. 9.

${ }^{4}$ Ibidem.

${ }^{5}$ Ibidem. 
las públicas y las privadas. Las primeras provenientes del sector público que se derivan de la acción de los poderes públicos, y las privadas que proceden del sector privado de quienes desempeñan tareas vinculadas a la atención de la salud pública. Esta disquisición se realiza sin el interés de ingresar en la discusión de la dicotomía de lo público y de lo privado y simplemente con el objetivo de ordenar metodológicamente tres conceptos fundamentales que están implicados en este estudio y que son la bioética, el bioderecho (o lo biojurídico) y la salud pública.

Por otro lado, la "cuestión moral" como una característica y concepción humana que sujeta las acciones personales del individuo per se o a través de las instituciones, involucra entre otras cosas la necesidad de una "pesquisa" de lo que se debe hacer, es decir una búsqueda, ${ }^{6}$ que apunta a definir las conductas a seguir y las que no, en función de procesos de valoración. Este concepto abierto de la moral, en contraposición a otros significados que aluden a códigos preexistentes y dogmáticos, parece más adecuado, pues abre posibilidades de observación y discusión de criterios de valoración en forma ampliada, permitiendo recrear nuevas conductas, a la luz de los comportamientos humanos individuales y las tendencias globales actuales.

De esta forma, la bioética vinculada a una pesquisa de orden moral es llamada a generar nuevos ámbitos de debate y respuestas a nuevos problemas que están continuamente gestándose a escala global y que de un modo $u$ otro involucran la salud humana y la salud pública, dos conceptos que deben ser diferenciados. La salud humana integra el elenco de la categoría de los derechos humanos ampliamente reconocida en múltiples instrumentos jurídicos de rango internacional, quizás los más paradigmáticos sean la Declaración Universal de Derechos Humanos (1948) y la Convención Americana sobre Derechos Humanos (Pacto de San José, Costa Rica, 1969).

${ }^{6}$ Ibidem. 
Con mayor actualidad el derecho a la salud, constituye un corolario de la dignidad humana fundamento de los ordenamientos jurídicos nacionales, recogido en ciertos casos con mayor énfasis expresamente en las cartas constitucionales como es el caso del derecho brasileño siguiendo la orientación del derecho alemán.

Por su parte la salud pública también recogida en las Constituciones como deber del Estado y desarrollada en innúmeras normas inferiores de carácter reglamentario, involucra además de cuestiones de política pública y burocracia, otros conceptos alusivos a las técnicas, métodos y prácticas terapéuticas orientadas hacia la población local. Pero también y desde un punto de vista político y jurídico la salud pública, representa uno de los mayores desafíos a nivel regional y mundial, en particular por ciertos factores supervinientes, como las crecientes migraciones por causas económicas, sociales y políticas o bien por desastres naturales.

Tanto en el caso del deber del Estado de proteger la salud de su población, como en el supuesto de la salud pública considerada objetivo prioritario de la agenda internacional, son necesarias el desarrollo de políticas públicas locales y globales orientadas a satisfacer las necesidades e intereses de carácter colectivo y la mejora en la calidad de vida de las personas, junto a la atención de urgencias sanitarias como las epidemias o pandemias. En concreto la salud pública trata del cuidado de los derechos colectivos e intereses difusos, que repercuten en el derecho/deber de cuidar de la salud humana.

Definiciones de orden sociocultural y político y en particular decisiones políticas que involucran el desarrollo de nuevas tecnologías generan una serie de conflictos de orden bioético y biojurídico, algunos de los cuales serán analizados sin que deba entenderse agotado el elenco de los mismos. El contexto de análisis se dispone en primer lugar en el terreno de la bioética, estrechamente relacionada e influenciada por la moral en la definición lisa y llana de moral de "lo que está bien y lo que no". Por 
otra parte el bioderecho también juega un rol preponderante ya que de diversas formas a través de la dogmática jurídica, abriga conceptos morales y éticos como fundamentos de los derechos humanos individuales y colectivos en general y en particular de referencia a la salud pública entendida como un subsistema jurídico en el que convergen acciones públicas y privadas, nacionales e internacionales, con el objetivo de dar satisfacción a necesidades e intereses de orden colectivo, pero que indirectamente impactan en los derechos individuales, colectivos y de futuras generaciones.

\section{PRINCIPALES PROBLEMAS DE LA SALUD PÚBLICA GLOBAL Y SU RELACION CON LA BIOÉTICA Y EL BIODERECHO}

Los procesos de globalización socioculturales y especialmente los de mercado impactan en la salud pública a escala global y local; las crisis financieras y sanitarias se reflejan en la necesidad de uniformizar ciertas soluciones científico-técnicas, originadas generalmente en propuestas provenientes de los países desarrollados y muy particularmente de los considerados polos tecnológicos. El primer problema que salta a la vista es que las necesidades sanitarias y económicas de los países desarrollados y de su industria y mercado tecnológico distan mucho de las de los países en vías de desarrollo. La consecuencia de esta disparidad de intereses y necesidades es que lejos de solucionar los problemas sanitarios locales se generan costos de adquisición de productos muchas veces innecesarios, con riesgos para la salud de la población y para el orden económico nacional, principalmente para los sectores tecnológicos y farmacéuticos locales y para el Estado.

En función de lo expresado cabe agregar algunas cuestiones actuales de carácter conflictivo en el ámbito de la salud pública global y que involucran el orden bioético y biojurídico: a) las que denominamos "cues- 
tiones de género" y consideramos únicamente las atinentes a las mujeres, por ser las más visiblemente perjudicadas en los aspectos que se observan; b) la ética profesional y la objeción de conciencia; c) la insuficiencia de la participación ciudadana y la difícil construcción de modelos de gobernanza capaces de influir en las decisiones de la industria tecnológica, así como en la construcción de políticas públicas tanto en materia del uso de la tecnología como de salud pública; d) la producción sin restricción de técnicas sin ninguna consideración ética sobre los impactos en las generaciones humanas futuras; e) la normalización técnica y su relación con las normas jurídicas; e) las razones de justicia social.

La discriminación en cuanto fenómeno de origen cultural ha minado todas y cada una de las disciplinas científicas, y también la medicina y el derecho. Como bien afirman Débora Diniz y Dirce Guillem, "el reconocimiento de que los papeles y las definiciones de lo masculino y lo femenino eran resultados de elecciones y preferencias socioculturales”, sembró un desconcierto en muchas áreas del conocimiento. ${ }^{7} \mathrm{El}$ resultado a partir de la toma de conciencia del origen de la discriminación fue favorable considerando, nuevos enfoque científicos y tecnológicos desarrollados a partir de este hecho. En efecto la sensibilización profesional en la salud se debió a los estudios de género llevados a cabo ${ }^{8}$, sumado a las grandes movilizaciones y reclamos de mayor justicia social basados en la necesidad de diferenciación en los procedimientos utilizados en la medicina, tanto en las etapas de diagnóstico como de tratamientos, orientados especialmente a la curación de enfermedades y epidemias de alto impacto en la población femenina como el HIV, o los cánceres de útero y de mama. Los avances en el reconocimiento de los derechos de las mujeres permitieron cambios en

${ }^{7}$ Diniz, Deborah; Guillem, Dirce. Bioética e Gênero. In: Fabri dos Anjos, Márcio; De Siqueira, José Eduardo (Orgs.). Bioética no Brasil, tendências e perspectivas. São Paulo: Sociedade Brasileira de bioética; Ideias Letras, 2007. p. 95-112. (Traduc. propia).

8 Ibidem. 
la investigación científica teniendo en cuenta las características orgánicas y estructurales propias de las mujeres, asociadas también a las condiciones socioculturales.

Sin embargo a medida que las nuevas tecnologías avanzaban junto a la experimentación, surgían otros problemas que directamente afectan los derechos de las mujeres. Son los casos del uso de las técnicas de reproducción asistida, la experimentación con embriones humanos y la legalización del aborto con la comercialización de drogas autorizadas cuyo uso es jurídicamente permitido durante las primeras semanas de gestación.

La discusión fundamental sobre el aborto que se basa en la determinación de la existencia o no de vida humana del no nacido, continúa siendo un quid de índole moral, ético y jurídico, con las consiguientes discordancias dentro de un mismo ordenamiento jurídico en que el no-nacido tiene derechos reconocidos de carácter hereditario y el aborto es legal. Pero la cuestión no es tan fácil de resolver desde la pregunta que precede toda discusión ética sobre el aborto que como señala Javier Gafo, ${ }^{9}$ suele formularse de la siguiente manera: “¿Cuándo comienza la vida humana en el desarrollo embrionario?, o ¿desde cuándo existe un ser humano o una persona humana? Aún cuando el Derecho adopte una respuesta y ello contribuya a la despenalización y o legalización del aborto, ello no resuelve la totalidad de conflictos a nivel de salud pública que se presentan. Puede parecer innovador el sistema jurídico uruguayo que despenaliza el aborto y lo autoriza si se cumplen las condiciones establecidas en la ley nro. 18.987, de 22 de octubre de $2012^{10}$, entre otras la de encontrarse dentro de las doce primeras semanas de gravidez, acudir al Sistema Nacional integrado de Salud y recibir apoyatura interdisciplinaria debiendo manifestar las circunstancias de las condiciones de la gravidez y las causas por las

${ }^{9}$ Gafo Javier. 10 Palabras clave en bioética. Navarra: Editorial Verbo Divino, 2000. p. 50.

${ }^{10}$ Publicada DO, 29.11.2012. 
que se desea interrumpir el embarazo; una vez cumplidos una serie de procedimientos y manifestado el consentimiento de la mujer, se autoriza el acto médico considerado sin valor comercial.

Por otra parte la propia ley nro. 18.987/2012, prevé una serie de deberes de los profesionales integrantes del equipo los cuales refieren en términos generales a apoyar a la mujer, orientándola y asesorándola sobre la prevención de futuros embarazos, el acceso a los medios y a programas de planificación familiar, garantizando que la decisión de la misma esté exenta de presiones de terceros, y debiéndose abstener el equipo profesional actuante de la función de denegar o autorizar la interrupción de la gestación. Más aún la norma regula la objeción de conciencia aplicable a los médicos ginecólogos y el personal de salud que intervengan en los procedimientos, manifestación que podrá revocarse en forma expresa en cualquier momento, entendiéndose revocación tácita la participación en los procedimientos; no pudiendo negarse a realizar dichos procedimientos los profesionales que no hayan expresado dicha objeción.

Sin embargo y a pesar de todas las garantías mencionadas, la objeción de conciencia que puede definirse como el no cumplimiento o acatamiento de la norma jurídica por razones de libertad de pensamiento y de creencias fundadas en motivaciones éticas, religiosas o morales entre otras causas $^{11}$, puede transformarse en un escollo difícil de sortear para los objetivos de la ley. Como bien señalan Deborah Diniz y Dirce Guillerme, ${ }^{12}$ la universalidad del derecho de objeción de conciencia no es un riesgo para los servicios de la salud, pues en general es utilizado en forma particular,

${ }^{11}$ Javier Juvenal, M. Reflexiones sobre la objeción de conciencia e ideario en el Uruguay a partir de las leyes 18.987 y 18.473. In: Blengio Valdés, Mariana (Coord.). Revista de Derecho Público, Montevideo, año 22, n. 43,2013, p. 33-56. Disponible en: <http://www.revistaderechopublico.com.uy/>. Acceso en: 27 oct. 2014.

${ }^{12}$ Diniz, Deborah; Guillem, Dirce. Bioética e Gênero. In: Fabri dos Anjos Márcio; De Siqueira, José Eduardo (Orgs.). Bioética no Brasil, tendências e perspectivas. São Paulo: Sociedade Brasileira de bioética; Ideias Letras, 2007. p. 95-112. (Trad. propia). 
es decir ante supuestos concretos individuales, pero -y en tal caso podría bajo ciertas características llegar a interpretarse como manifestación del fenómeno discriminatorio hacia las mujeres como género- existe el fenómeno de generalización de la objeción de conciencia institucionalizada. Es el supuesto citado por las autoras cuando, "la objeción de conciencia asciende a la esfera pública y que en general, son situaciones que envuelven mujeres y decisiones públicas”, siendo la situación más corriente la comentada, lo que promueve el aborto ilegal en condiciones tales que se acude a la atención del servicio de salud cuando ya existe riesgo de vida. ${ }^{13}$ En estos casos la confrontación de un derecho individual como es el de la objeción de conciencia ejercido en forma colectiva, atenta contra el derecho individual de la mujer poniendo en riesgo derechos colectivos de género y también el sistema de salud pública.

La ausencia de modelos de participación y de gobernanza capaces de influir en los gobiernos y sus decisiones con el fin de reorientar la tecnología hacia la satisfacción del interés general, sin que por ello se vea perjudicado el lucro de la empresa, es otro de los conflictos visibles de orden bioético y biojurídico que impactan en la salud pública. La consecuencia inicial es que grandes cantidades de recursos, tanto de capacidades como de dineros públicos y privados, se destinan a la creación de innovación tecnológica que no satisface el interés general, entendido este concepto como las necesidades actuales y futuras de la población local y global.

Este fenómeno se ve reflejado claramente en el manejo de las tecnologías respecto del tratamiento de las epidemias y pandemias. El virus del ébola fue descubierto hace más de tres décadas pero aún no se ha creado una vacuna eficiente, a pesar de las diversas aplicaciones biotecnológicas en la medicina. Igual observación merece el tratamiento de la malaria y el dengue. Es definitiva se trata de casos de injusticia social en

${ }^{13}$ Ibidem. (Trad. propia). 
que la distribución de la renta global y el modo inequitativo de inversión en innovación tecnológica no responde a las necesidades de la mayor parte de la población cuya salud se ve afectada. Por el contrario la reacción de parte del sector tecnológico y político aparece ante la posibilidad cierta de una pandemia de ébola que afecte a los países desarrollados también, lo que motivó un brusco cambio de ruta de la innovación tecnológica que ensaya actualmente una vacuna y la adopción de medidas a escala global con la promoción de la OMS, la UE y la Ocde entre otros organismos internacionales.

Pero además la participación ciudadana en las políticas de salud pública reducen la distancia que existe entre la técnica y el ciudadano.

Además de los riesgos que dichas tecnologías encierran desde el punto de vista comercial y social, existen otros que vinculan los referentes a los ciudadanos como consumidores finales pues los métodos y técnicas médicas innovadoras son aplicados en la población en forma más o menos generalizada con escasa información para los ciudadanos. En particular y con frecuencia pacientes en difíciles circunstancias de salud, son informados que deben prestar su consentimiento (informado) sobre técnicas cuyos procedimientos y efectos le son desconocidos. La duda que surge ante la sofisticación de las técnicas y métodos clínicos utilizados por la medicina, es si realmente se cumple el principio de autonomía que permite a la persona en uso de su capacidad escoger entre las diversas opciones que el profesional le presenta en forma voluntaria y libre.

De esta forma aquí también se observan incongruencias de los ordenamientos jurídicos, respecto de las mismas técnicas con diversas aplicaciones. Mientras que para las aplicaciones biotecnológicas en la agricultura prácticamente todos los sistemas jurídicos del mundo han dado y profundizado un importante debate sobre los riesgos para la salud y el medio ambiente, y la necesidad de garantizar la participación ciudadana, absurdamente las mismas biotecnologías son utilizadas con fines terapéuti- 
cos humanos, a través de tratamientos y medicamentos, en el más absoluto sigilo pudiendo generar los mismos riesgos tanto para el ambiente, como para la salud humana.

La importancia de este conflicto planteado abarca las presentes pero también las futuras generaciones humanas, por los posibles riesgos principalmente al medio ambiente y a la salud humana. Las preocupaciones éticas, políticas y jurídicas por la futura humanidad ha sido recogida en innúmeros convenios internacionales, como la Declaración de Río de la Convención sobre Desarrollo Humano y Medio Ambiente (CNUMAD, 1992) y muy particularmente en la Declaración Universal sobre el Genoma Humano y los Derechos Humanos. Un referente jurídico importante, lo constituye la Constitución Federal de Brasil, que ubica el Capítulo IV "De Ciencia y Tecnología”, en el Título VIII correspondiente al Orden Social y no dentro del Orden Económico y Financiero, lo que significa que la orientación prioritaria debe ser social. El artículo 218 parágrafo 2 de la Constitución Federal es una norma compleja y completa, en el sentido que asocia los fines sociales y productivos a escala local y global, pues establece que la investigación tecnológica se dirigirá preponderantemente a la solución de los problemas brasileños y para el desarrollo de sistema productivo nacional y regional.

En definitiva la cuestión de la sustentabilidad de la innovación tecnológica, implica por sobre todo la adecuada gestión de los riesgos derivados de dichas actividades tecnológicas que impactan en el medio ambiente y en la salud pública, sin perjuicio de la promoción de la innovación pero orientada a la resolución de la problemática social con énfasis particular en la salud pública, la industria farmacéutica, alimenticia, la vivienda y la satisfacción de las principales necesidades humanas.

Otras disposiciones que se ajustan a este enfoque son el artículo 225 de la Constitución Federal del Brasil, que consagra el derecho al medio ambiente ecológicamente equilibrado para una sana calidad de vida, 
y la ley nro. 6.938/81 (Política Nacional de Medio Ambiente) que establece en su artículo 2 el destino de los incentivos al estudio y a la investigación para su uso racional y la protección de los recursos naturales.

Pero además la importancia de una ciudadanía capaz de incidir en las decisiones políticas y socioeconómicas de una nación, se relaciona con otro orden de conflictos derivados de desvíos reglamentarios en los sistemas de ordenación técnica a través de los estándares de calidad. Es fundamental en estos casos la participación de ciudadanos calificados integrando órganos estatales principalmente los comités técnicos. En general, sistemas como la Unión Europea y el brasileño y progresivamente todos los sistemas jurídicos prevén la integración de dichos comités por personas de notorio destaque tanto en valores (éticos) como en idoneidad científico-académico. Si bien gran parte de los procesos de normalización técnica se dan en el ámbito privado de la industria tecnológica, cada vez más y ante la necesidad de procesos de gestión de riesgos, se prevé la conformación de estos comités en el ámbito público con amplia integración a modo de democratizar y dar transparencia tanto a las decisiones como a los controles y niveles de calidad exigidos a las industrias tecnológicas. Dichos comités, órganos públicos o privados, cuentan con potestad reglamentaria, de policía y disciplinaria.

Las decisiones sobre la adopción de medidas de gestión de riesgos, residuos, sistemas de emergencia y otros factores de impacto en la salud pública, son materia de su competencia. Las normas técnicas dictadas por estos comités técnicos, usualmente adquieren efecto vinculante por ser incorporados a reglamentos en el mejor de los casos y cuando dichos comités son personas privadas de derecho público o integran el Estado en sentido restrictivo. Por otra parte se cumplen procesos de información y consulta pública a la ciudadanía, lo que no significa necesariamente que sea una garantía para el sistema de salud pública, teniendo en cuenta la distancia de las materias que se discuten y la información calificada 
que no siempre es decodificada para la población media como forma de garantizar el derecho a la participación y la libre elección de los métodos terapéuticos.

En conclusión el impacto del ciudadano común a través de su participación y en la evaluación de riesgos tecnológicos a través de los procedimientos de consulta pública, es de una escasa incidencia en la reorientación del mercado de la innovación tecnológica aplicada a la salud pública; ello involucra además la ausencia de discusión de aspectos bioéticos y cuestiones definitivamente técnicas como es el criterio de equivalencia, que implica la autorización de fármacos y otros productos como las vacunas, basados en pruebas y utilizadas en poblaciones condicionadas por factores climáticos diversos y de otro orden, y que en definitiva ponen en duda la legitimidad de dicho criterio tanto en términos científicos y tecnológicos como jurídicos, políticos y bioéticos.

La justicia social como resultado más que como ideal continúa siendo el objetivo y desde ese enfoque cabe hacer referencia a dos situaciones en particular; la primera de ellas es la falta de acceso a los beneficios y avances médicos actuales por buena parte de la población mundial en igualdad de condiciones con quienes se ven beneficiados, lo que supone como se ha dicho ya, una distribución justa y equitativa de la renta global, reflejada en la transferencia tecnológica de los países desarrollados a los demás que por un motivo u otro es de difícil cumplimiento aún cuando contradiga la universalidad de los servicios de salud pública.

La otra situación que escogemos referir entre tantas otras es el de la experimentación animal y el impacto que ello causa en la sensibilidad ciudadana y que en ciertos sistemas ha llevado a regular mediante leyes de protección animal ciertas prácticas y prohibir otras. Es necesario como sugiere Martha C. Nussbaum, ${ }^{14}$ superar ciertas barreras de la clá-

${ }^{14}$ Nussbaum, Martha C. Fronteiras da justiça. São Paulo: Martins Fontes, 2013. p. 412. 
sica teoría de la justicia de John Rawls basada en su ordenación conforme a las ideas kantianas y del contrato social, ingresando a otros contextos capaces de vincular otras capacidades, concretamente incorporando los animales, lo que permite abrir la discusión de lo que es justo o no para otros seres vivos, más allá de los humanos, con el fin de dignificarlos en el respeto a su naturaleza como muy bien señala M. Nussbaum.

Una teoría de la justicia ampliada implica también un repensar las formas sobre cómo se evalúan los riesgos tecnológicos, y la determinación del quantum de aceptación de los mismos que como bien lo explica José Heck ${ }^{15}$ implica interrogarnos acerca de si además de los hombres debemos tomar en cuenta para la determinación de la magnitud del riesgo otras entidades, como los animales.

No quedan fuera tampoco los conflictos bioéticos y de bioderecho pero vinculados a los “tipos de determinismo" ya sean de orden físico, social o sicológico. Al respecto cabe citar la alerta de Carlos Santiago Nino, ${ }^{16}$ en referencia al determinismo sicológico defendido por quienes afirman que, "las acciones humanas están condicionadas por sucesos, inclinaciones tendencias, etc., de índole mental". Nuevos brotes científicos de determinismo y sus efectos en la sociedad tienen su origen en las más diversas experiencias biotecnológicas y principalmente basadas en el estudio de los genes de quienes delinquen en forma contumaz, con el fin de establecer anticipadamente hechos en lo que constituye una práctica de discriminación inaceptable de todo punto de vista humano, no sólo ético y jurídico.

\footnotetext{
${ }^{15}$ Heck, José. Bioética: Autopreservación, enigmas y responsabilidad. Florianópolis, Ed. UFSC, 2011. p. 55.

${ }^{16}$ Nino, Carlos Santiago. Ética y Derechos humanos. 2. ed. Buenos Aires: Ed. Astrea, 2007. p. 267-269. Sobre el punto se destaca el análisis del destacado autor que realiza en su obra acerca del principio de dignidad de la persona humana, la adscripción a la responsabilidad y el determinismo en sus diversas modalidades.
} 
Por último la complejidad de la problemática bioética nos induce a vincular los conflictos derivados desde los derechos humanos con los conceptos de democracia local y global. Si la bioética es como afirma Joaquim Clote $^{17}$ una disciplina ocupada del uso correcto de las nuevas tecnologías en el área de las ciencias médicas con el fin de dar solución adecuada a los dilemas de índole moral que se le presentan, no cabe ningún tipo de argumentación para apartarse de las bases de dicha disciplina.

Las bases de la bioética y el bioderecho no deberían jamás estar superadas por normas técnicas de carácter general, o buenas prácticas cuya vigencia depende de la próxima innovación tecnológica. Más aún, ni la generalización de ciertos principios es capaz de resolver los conflictos antes planteados, y lejos de hacerlo genera otros, pues como señala Joaquim Clote ${ }^{18}$ el Convenio de Derechos Humanos y Biomedicina del Consejo Europeo, advierte sobre el conflicto del pluralismo ético de nuestra sociedad global y la dificultad que se establece a la hora de unificar criterios sobre el bien y el mal, lo justo y lo injusto, lo correcto e incorrecto a la hora de aprobar conductas de impacto en el bienestar de las personas, consideradas en la esfera individual o colectiva.

\section{CONCLUSIONES}

Los modelos democráticos futuros basados en concepciones igualitarias por encima de los tradicionales derechos humanos, y concretamente bajo la confluencia del genoma humano como "unidad fundamental de todos los miembros de la familia humana” (Art. 1, Declaración sobre el Genoma Humano y los Derechos Humanos), llaman a la reflexión a lo que está por debajo y por dentro de los conflictos bioéticos y biojurídicos actuales y futuros.

${ }^{17}$ Clote Joaquim. Bioética uma aproximação. Porto Alegre: EDIPUCRS, 2003. p. 33.

${ }^{18}$ Ibidem, p. 128-129. 
Se percibe la insistencia en la discriminación de las personas por razones de género, etnia, cultura, y condiciones socioculturales y económicas, mediante nuevas formas de discriminación, que surgen de la mano de los avances tecnológicos y recaen en los sectores más vulnerables de la población mundial con mayor visibilidad que antaño. Concepciones totalitarias emergentes capaces de alentar innovaciones tecnológicas con el fin de violentar sectores de la población más vulnerables, son también objeto de preocupación social y política.

Por ello el debate se reconduce a nuestro modo de ver, en el análisis del equilibrio entre dos esferas complementarias: a) la permanencia de la dignidad humana, concepto dinámico y cuyo mínimo contenido irreductible garantiza su atemporalidad; eje conductor para la observación y resolución de los dilemas de orden moral y ético; y b) la revaloración de la soberanía de los Estados, enfocada en la defensa de los derechos de autodeterminación de los pueblos y la libre elección de su destino, sus valores políticos, sociales, culturales y económicos.

\section{REFERENCIAS}

CARVALHO FORTES, Paulo Antonio; CAMPOS PAVONE ZOBOLI, Elma Lourdes. Bioética e saúde pública: entre o individual e o coletivo. In: CARVALHO FORTES, Paulo Antonio; CAMPOS PAVONE ZOBOLI, Elma Lourdes (Orgs.). Bioética e saúde pública. São Paulo: Edições Loyola, 2003.

CLOTE, Joaquim. Bioética uma aproximação. Porto Alegre: EDIPUCRS, 2003. DINIZ, Deborah; GUILLEM Dirce. Bioética e gênero. In: FABRI DOS ANJOS, Márcio; DE SIQUEIRA, José Eduardo (Orgs.). Bioética no Brasil, tendências e perspectivas. São Paulo: Sociedade Brasileira de Bioética; Ideias Letras, 2007. DURANT, Guy. A bioética, natureza, princípios, objetivos. São Paulo: Paulus, 1995. 
GAFO, Javier. 10 Palabras clave en bioética. Navarra: Editorial Verbo Divino, 2000 .

HECK, José. Bioética: autopreservação, enigmas y responsabilidad. Florianópolis, Ed. UFSC, 2011.

JAVIER, Juvenal M. Reflexiones sobre la objeción de conciencia e ideario en el Uruguay a partir de las leyes 18.987 y 18.473. In: BLENGIO VALDÉS, Mariana (Coord.). Revista de Derecho Público, Montevideo, año 22, n. 43, 2013. Disponible en: <http://www.revistaderechopublico.com.uy/>. Acceso en: 27 oct. 2014. NINO, Carlos Santiago. Ética y Derechos humanos. 2. ed. Buenos Aires: Ed. Astrea, 2007.

NUSSBAUM, Martha C. Fronteiras da justiça. São Paulo: Martins Fontes, 2013.

Recebido em: 28/5/2015

Aceito em: 2/5/2015 